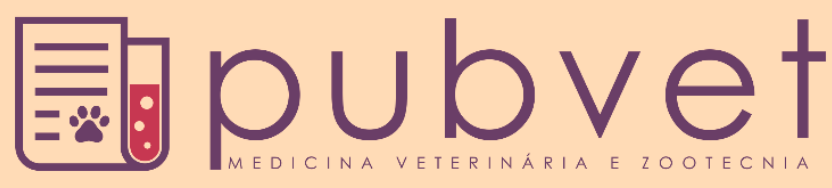

https://doi.org/10.31533/pubvet.v14n2a511.1-4

\title{
Uso da medicina integrativa na reabilitação de felino com lesão óssea: relato de caso
}

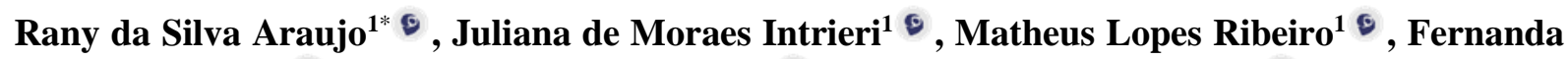 \\ Mattoso Jácome $^{1 \bullet}$, Agatha Campinho Belsito ${ }^{\circ}$, Magda Alves de Medeiros ${ }^{2}{ }^{\circ}$ \\ ${ }^{I}$ Alunalo de graduação da Universidade Federal Rural do Rio de Janeiro. Seropédica -RJ Brasil. \\ ${ }^{2}$ Professora da Universidade Federal Rural do Rio de Janeiro, Departamento de Ciências Fisiológicas. Seropédica -RJ Brasil. \\ *Autor para correspondência, E-mail: rany.silva.araujo@hotmail.com
}

\begin{abstract}
Resumo. Um felino, fêmea, sem raça definida e de aproximadamente três anos sofreu fratura traumática múltipla do fêmur esquerdo e foi submetida à cirurgia de osteossíntese no Hospital Veterinário de Pequenos Animais da Universidade Federal Rural do Rio de Janeiro. No período pós-cirúrgico, o paciente apresentou além de dor local, perda de propriocepção do membro e perda de sensibilidade das falanges distais. Quatro semanas após a cirurgia foi iniciado tratamento por acupuntura, moxabustão e terapia floral. Ao longo de cinco sessões o animal apresentou melhora da dor e da sensibilidade, restabelecendo a funcionalidade do membro e recuperando qualidade de vida. No presente estudo, a associação das diferentes técnicas da medicina veterinária integrativa pareceu diminuir o tempo de recuperação e facilitar a reabilitação de lesões ortopédicas com comprometimento de nervos periféricos.
\end{abstract}

Palavras chave: acupuntura, floral, fratura, moxabustão, recuperação

\section{Use of integrative medicine in the rehabilitation of feline with bone lesions: case report}

\begin{abstract}
A feline, female, of no breed and approximately three years old, suffered multiple traumatic fractures of the left femur and underwent osteosynthesis surgery at the Pet Veterinary Hospital of Universidade Federal Rural do Rio de Janeiro. In the postoperative period, the patient presented local pain, loss of proprioception of the limb and loss of sensation of the distal phalanges. Four weeks after the surgery, acupuncture, moxibustion and flower therapy treatment was started. Over five sessions, the animal improved pain and sensitivity, restoring limb functionality and restoring quality of life. In the present study, the association of different techniques of integrative veterinary medicine seemed to decrease recovery time and facilitate the rehabilitation of orthopedic lesions with peripheral nerve involvement.
\end{abstract}

Keywords: acupuncture, floral, fracture, moxibustion, recovery

\section{Medicina integrativa en la rehabilitación de felinos con lesión ósea: reporte de caso}

Resumen. Una gata, criolla y de aproximadamente tres años, sufrió múltiples fracturas traumáticas del fémur izquierdo y se sometió a una cirugía de osteosíntesis en el Hospital Veterinário de Pequenos Animais em la Universidade Federal Rural do Rio de Janeiro. En el postoperatorio, el paciente presentó dolor local, pérdida de propiocepción de la extremidad y pérdida de la sensación de las falanges distales. Cuatro semanas después de la cirugía, se 
inició el tratamiento con acupuntura, moxibustión y terapia floral. Durante cinco sesiones, el animal mejoró el dolor y la sensibilidad, restaurando la funcionalidad de las extremidades y restaurando la calidad de vida. En el presente estudio, la asociación de las diferentes técnicas de medicina veterinaria integrativa pareció disminuir el tiempo de recuperación y facilitar la rehabilitación de lesiones ortopédicas con afectación de nervios periféricos.

Palabras clave: acupuntura, fractura, floral, recuperación, moxibustión

\section{Introdução}

As fraturas femorais representam de $20 \%$ a $26 \%$ das fraturas em animais de companhia, sendo a maioria delas de origem traumática. Em um aspecto amplo, as fraturas podem ser entendidas como a interrupção da continuidade óssea e nesses casos, principalmente quando acomete a região femoral, a intervenção cirúrgica apropriada é indicada (Piermattei \& Flo, 2009).

A acupuntura é capaz de reduzir a inflamação, aumentando a circulação sanguínea local, e a dor através de mecanismos periféricos, espinhais e supraespinhais (Zhang et al., 2014), sendo utilizada na recuperação de fraturas (Nakajima et al., 2010). Pode ser associada com a técnica de moxabustão que tem como função aquecer e regular a circulação sanguínea e de energia, melhorando a cicatrização (Wegner et al., 2013). Os efeitos do trauma e da cirurgia podem ser tratados também pela terapia floral (Graham \& Vlamis, 1999). O objetivo deste trabalho é relatar o caso de fratura óssea em que foram utilizadas as três terapias supracitadas, correlacionando o uso da alopatia complementada com práticas da medicina integrativa na rotina médico-veterinária

\section{Material e métodos}

Uma gata fêmea, castrada, sem raça definida, de aproximadamente três anos e 5,3 quilos recebeu atendimento no Hospital Veterinário de Pequenos Animais da Universidade Federal Rural do Rio de Janeiro pela claudicação do membro pélvico esquerdo. $\mathrm{O}$ animal apresentava oligodipsia, anorexia e emagrecimento de aproximadamente 400 gramas em seis dias.

No exame radiográfico, observou-se fratura completa e dupla em terços proximal e médio do fêmur esquerdo, havia linhas de fratura em bisel, com severo desvio entre os segmentos e presença de ao menos uma esquírola visível (Figura 1). Notou-se moderado edema de tecido mole adjacente ao foco de fratura, sugerindo a presença de processo inflamatório. Diante deste quadro, recomendou-se a intervenção cirúrgica de osteossíntese do fêmur esquerdo mediante a inserção de pinos intramedulares e três cerclagens (Figura 2). Após a cirurgia foi prescrito Dipirona $25 \mathrm{mg} / \mathrm{kg} / \mathrm{SID}$ por sete dias, Cloridrato de Tramadol $1 \mathrm{mg} / \mathrm{kg} / \mathrm{SID}$ por sete dias, Meloxicam 0,1mg/kg/SID por 3 dias e Amoxicilina com Clavulanato $25 \mathrm{mg} / \mathrm{kg} / \mathrm{BID}$ por 10 dias, sendo estes administrados por via oral.

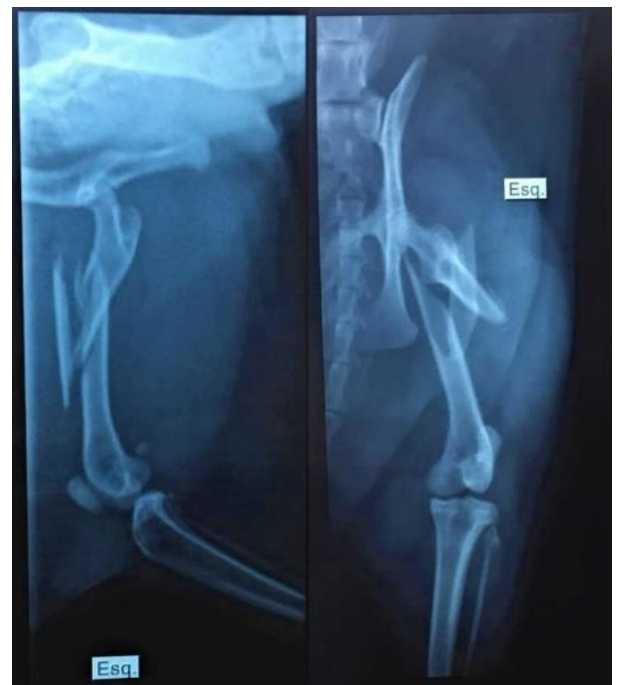

Figura 1. Exame radiográfico identificando fratura completa e dupla do fêmur esquerdo.

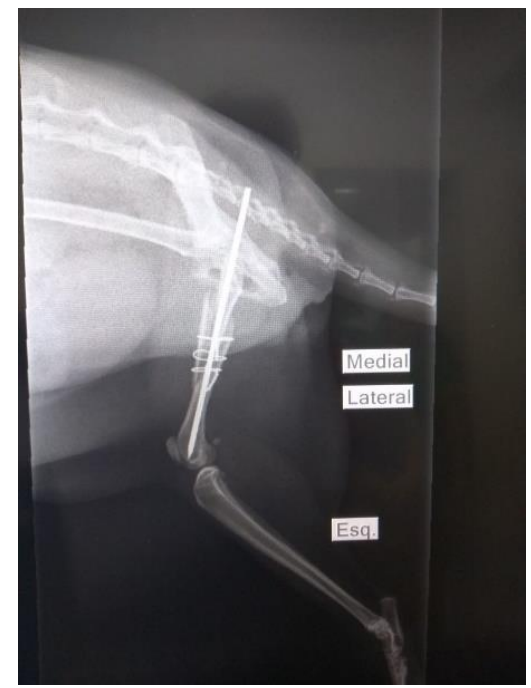

Figura 2. Exame radiográfico identificando a osteossíntese realizada. 
O retorno ocorreu três dias após a cirurgia, o animal demonstrava sinais de dor mesmo que o local acometido pela fratura não fosse manipulado. Aplicou-se $0,3 \mathrm{mg} / \mathrm{kg}$ de Metadona IM, 0,2 mg/kg de Cetamina IM e 0,2 mg/kg de Midazolan IM. Aplicou-se 12,5 mg/kg de Dipirona SC e 0,1 mg/kg/SID Maxicam SC. Tendo em vista evitar o estresse de manipulação de medicamentos por via oral no paciente, interrompeu-se o uso da Amoxicilina com Clavulanato e foi prescrito, via subcutâneo, 50 $\mathrm{mg} / \mathrm{kg} / \mathrm{SID}$ de Ceftriaxona durante cinco dias e, por via oral, $5 \mathrm{mg} / \mathrm{kg} / \mathrm{BID}$ de Gabapentina durante quinze dias.

Ao retornar dez dias após a cirurgia, observou-se que o animal não apoiava o membro para se locomover e apresentava déficit de propriocepção no membro pélvico esquerdo de grau leve. Apresentava edema na região proximal ao pino e na região caudal dos músculos da coxa. Foi realizada drenagem do seroma e exame de raio x, que apontou alterações da posição dos implantes e marcou o edema do membro. Prescreveu-se Hirudoid BID tópico nos locais edemaciados e o uso de compressas quentes e frias durante dez minutos. Vinte-e-um dias depois da cirurgia, o pino intramedular foi retirado devido à formação de seroma e da dor ocasionada pelo implante. Nesse momento, repetiuse a prescrição de Dipirona $25 \mathrm{mg} / \mathrm{kg} / \mathrm{SID}$ por sete dias, Cloridrato de Tramadol $1 \mathrm{mg} / \mathrm{kg} / \mathrm{SID}$ por sete dias, Meloxicam 0,1mg/kg/SID por 3 dias e Amoxicilina com Clavulanato 25mg/kg/BID por 10 dias, sendo estes administrados por via oral.

Três dias depois, houve uma diminuição do seroma e uma dor local na região proximal ao fêmur. A defecação e a ingestão de água e alimentos estavam normalizadas. Neste momento, encaminhou-se o animal para a acupuntura pelo déficit de propriocepção do membro, perda da dor superficial da região do joelho para a região mais distal - presença de uma dor profunda e ausência da sensibilidade na região das falanges distais.

Após passados trinta e um dias da realização da osteossíntese e três dias após o período prescrito para a medicação alopática citada anteriormente, iniciou-se o tratamento integrativo. A primeira sessão de acupuntura se deu trinta e um dia após a cirurgia e nesta ocasião o diagnóstico pela medicina tradicional chinesa foi estabelecido e se constituía em estagnação de Qi (energia) e Xue (sangue). Foram utilizadas agulhas inseridas em pontos responsáveis pela dispersão de umidade e calor, de estagnação de Qi/Xue e restabelecimento de fluxo de Qi nos canais e colaterais. Os pontos estimulados na primeira sessão foram: VB30, E36, E35, R1, F3 e Baihui (Xie \& Preast, 2007). Na segunda sessão observou-se uma melhora na locomoção do animal e foi dado prosseguimento no tratamento com os pontos da primeira sessão. Na terceira sessão houve uma melhora ainda mais significativa, com um maior apoio da pata traseira esquerda e manteve-se a estimulação desses mesmos pontos de acupuntura.

Além do agulhamento, na terceira sessão se iniciou o tratamento com a essência floral Star of Bethlemhem que objetiva combater os efeitos de choque causado por situações indesejáveis como acontecimentos traumáticos e operações cirúrgicas, reestabelecendo a mente, o emocional e/ou físico sendo administradas duas gotas quatro vezes ao dia (Graham \& Vlamis, 1999). Ao retornar, na quarta sessão, a tutora relatou que o paciente conseguiu descer a escada de casa, apesar de ainda apresentar uma leve claudicação. Nesta sessão, além do agulhamento seco nos pontos, foi utilizada a técnica de moxabustão. Nesta técnica um bastão de Artemísia aceso é aproximado da pele e movimentado suavemente entre os pontos. Na quinta sessão, foram estimulados os pontos B23, Bahui, R1 e VB30 e realizada novamente a técnica de moxabustão.

Após cinco sessões, o animal apresentou alívio do quadro de dor, retomando seu comportamento característico e retornou a exercer suas atividades ao conseguir utilizar o membro posterior esquerdo como apoio, recuperando assim sua qualidade de vida de forma satisfatória.

\section{Resultados e discussão}

A acupuntura tem sido empregada com sucesso para tratar problemas agudos e crônicos de dor, sendo muito utilizada em afecções osteomusculares (WHO, 2003). Além da acupuntura, a técnica da moxabustão tem a função de aquecer, ou seja, dissipar o frio, o vento e a umidade, concomitantemente a regular a circulação sanguínea e de energia, atuando também na redução da dor e inflamação e na cicatrização (Wegner et al., 2013). Os florais de Bach são capazes de promover o equilíbrio do estado 
emocional e são considerados seguros e eficazes para o tratamento da dor. As práticas da medicina integrativa como a acupuntura, moxabustão e terapia floral fornecem métodos capazes de acelerar, subsequente à cirurgia ortopédica, a promoção da saúde e a recuperação da qualidade de vida do animal (Thaler et al., 2009).

\section{Referências bibliográficas}

Graham, H. \& Vlamis, G. (1999). Remédios Florais de Bach para animais. São Paulo, Brasil: Editora Pensamento.

Nakajima, M., Inoue, M., Hojo, T., Inoue, N., Tanaka, K., Takatori, R. \& Itoi, M. (2010). Effect of electroacupuncture on the healing process of tibia fracture in a rat model: a randomised controlled trial. Acupuncture in Medicine, 28(3):140-143.

Piermattei, B. D. L. \& Flo, G. L. (2009). Ortopedia e tratamento das fraturas dos pequenos animais (3 ed.). São Paulo: Editora Manolo.

Thaler, K., Kaminski, A., Chapman, A., Langley, T. \& Gartlehner, G. (2009). Bach Flower Remedies for psychological problems and pain: a systematic review. BMC Complementary and Alternative Medicine, 9(1):16.

Wegner, F., Costa, A. D., Ribeiro, G. K. S., Andofato, K. M. \& Comparin, K. A. (2013). Moxabustão: Uma Revisão da Literatura. Fiep Bulletin, 83.

WHO. (2003). World Health Organization. Diet, nutrition and the prevention of chronic diseases. Report of a joint WHO/FAO expert consultation. WHO technical report series, 916.

Xie, H. \& Preast, V. (2007). Xie's veterinary acupuncture: Wiley Online Library.

Zhang, R., Lao, L., Ren, K. \& Berman, B. M. (2014). Mechanisms of acupuncture-electroacupuncture on persistent pain. Anesthesiology: The Journal of the American Society of Anesthesiologists, 120(2):482-503.

Recebido: 26 de setembro, 2019.

Aprovado: 19 de novembro, 2019.

Publicado: 23 de março, 2020.

Licenciamento: Este artigo é publicado na modalidade Acesso Aberto sob a licença Creative Commons Atribuição 4.0 (CC-BY 4.0), a qual permite uso irrestrito, distribuição, reprodução em qualquer meio, desde que o autor e a fonte sejam devidamente creditados. 